\title{
Preferential recruitment of neutrophils by endothelin-I in acute lung inflammation induced by lipopolysaccharide or cigarette smoke
}

\author{
Tapan Bhavsar \\ Xing Jian Liu \\ Hardik Patel \\ Ralph Stephani \\ Jerome O Cantor \\ St John's University, School \\ of Pharmacy and Allied Health \\ Sciences, New York, USA
}

Correspondence: Jerome Cantor St John's University (SAH SB-30), 8000 Utopia Pkwy, Jamaica, NY I I439, USA

Tel +I 7189907495

Email jocantor@pol.net

\begin{abstract}
This study examined the role of endothelin-1 (ET-1) in recruiting inflammatory cells to the lung after induction of injury with either lipopolysaccharide (LPS) or cigarette smoke. Hamsters injected with either ET-1 or its precursor peptide (Big ET-1) prior to treatment with LPS or cigarette smoke had markedly increased concentrations of neutrophils in bronchoalveolar lavage fluid (BALF) despite a reduction in total numbers of BALF leukocytes. Furthermore, the effect of ET-1 on smoke-exposed animals was reversed by addition of an endothelin-A receptor antagonist. These results are consistent with preferential recruitment of neutrophils by ET-1, and suggest that inhibition of this proinflammatory mediator may decrease acute pulmonary inflammation associated with cigarette smoke and other pulmonary toxins.
\end{abstract}

Keywords: endothelin, lipopolysaccahride, cigarette smoke, neutrophils, lung

\section{Introduction}

Endothelin-1 (ET-1) is a proinflammatory mediator that is formed by the conversion of a 39-amino acid precursor peptide, Big ET-1, to the 21-amino acid peptide, ET-1 (Hisaki et al 1994; Laporte and Sirois 1997; Fagan et al 2001). It activates neutrophils and induces the release of a variety of cytokines by monocytes (Teder and Noble 2000). In addition, ET-1 is a potent vasoconstrictor that plays an important role in ischemic injury (Hassan et al 1997; Aguilar et al 2000; Park and Thornhill 2001).

Previously, this laboratory has shown that inhibition of ET-1 activity with either phosphoramidon (an endothelin-converting enzyme inhibitor) or an endothelin receptor antagonist markedly reduces lipopolysaccharide (LPS)-induced influx of neutrophils into the lung (Bhavsar et al 2008). However, it remains to be determined whether ET-1 acts selectively on neutrophils or has a wider role in the recruitment of leukocytes.

To address this question, we examined the effect of exogenously administered ET-1 or its precursor peptide, Big ET-1, on LPS-induced inflammation in hamster lungs. Five hours after LPS instillation (when the inflammatory reaction was maximal), the animals were sacrificed and bronchoalveolar lavage fluid (BALF) leukocytes were examined. The results indicate that both ET-1 and Big ET-1 induce a preferential influx of neutrophils into the lung.

Since cigarette smoke contains significant amounts of LPS (Hasday et al 1999; Larsson et 2004), we also investigated the possibility that ET-1 might produce a similar outcome in smoke-exposed animals. Measurement of BALF leukocytes again demonstrated a selective recruitment of neutrophils by ET-1. Furthermore, this effect was reversed by concomitant treatment with an endothelin receptor antagonist, suggesting a possible role for this type of agent in reducing smoke-related lung injury. 


\section{Methods}

\section{Experimental design}

LPS model

Female Syrian hamsters, weighing approximately $100 \mathrm{~g}$ each, were divided into 4 groups as follows: Group 1 was treated intraperitoneally (IP) with $20 \mu \mathrm{g}$ bovine "big" ET-1 (Sigma-Aldrich, St Louis, MO) in $0.2 \mathrm{~mL}$ PBS, followed $60 \mathrm{~min}$ later by $10 \mu \mathrm{g}$ intratracheal (IT) LPS (Sigma-Aldrich) in $0.2 \mathrm{~mL}$ PBS; Group 2 was treated IP with $20 \mu \mathrm{g}$ of human ET-1 (Sigma-Aldrich) in $0.2 \mathrm{~mL}$ PBS, followed 60 min later by $10 \mu \mathrm{g}$ IT LPS in $0.2 \mathrm{~mL}$ PBS; Group 3 was treated IP with $0.2 \mathrm{~mL}$ PBS, followed $60 \mathrm{~min}$ later by $10 \mu \mathrm{g}$ IT LPS in $0.2 \mathrm{~mL}$ PBS; Group 4 was treated IP with $0.2 \mathrm{~mL}$ PBS, followed $60 \mathrm{~min}$ later by $0.2 \mathrm{~mL}$ IT PBS. All animals were sacrificed $5 \mathrm{~h}$ after LPS instillation, when the pulmonary inflammatory reaction was maximal, and both the total number of BALF leukocytes and percentage of BALF neutrophils were measured (Nadkarni et al 2005). The protocol was approved by the institutional animal care committee.

\section{Cigarette smoke model}

Female Syrian hamsters, weighing approximately $100 \mathrm{~g}$ each, were divided into 4 groups as follows: Group 1 was treated IP with: 1) $500 \mu \mathrm{g}$ of HJP272, a novel endothelin-A receptor antagonist, in $0.5 \mathrm{~mL} \mathrm{Na}_{2} \mathrm{CO}_{3}$ and 2) $5 \mu \mathrm{g}$ ET-1 in $0.25 \mathrm{~mL}$ PBS $60 \mathrm{~min}$ later, followed immediately by exposure to cigarette smoke for $2 \mathrm{~h}$. This regimen was continued for 3 consecutive days; Group 2 was treated IP with: 1) $0.5 \mathrm{~mL} \mathrm{Na}_{2} \mathrm{CO}_{3}$ and 2) $5 \mu \mathrm{g}$ ET-1 in $0.25 \mathrm{~mL}$ PBS 60 min later, followed immediately by exposure to cigarette smoke for 3 consecutive days as per Group 1; Group 3 was treated IP with: 1) $0.5 \mathrm{~mL} \mathrm{Na}_{2} \mathrm{CO}_{3}$ and 2) $0.25 \mathrm{~mL}$ PBS $60 \mathrm{~min}$ later, followed immediately by exposure to cigarette smoke for 3 consecutive days as per Group 1; Group 4 was treated IP with: 1) $0.5 \mathrm{~mL} \mathrm{Na}_{2} \mathrm{CO}_{3}$ and 2) $0.25 \mathrm{~mL}$ PBS 60 min later, followed by exposure to room air for 3 days. All animals were sacrificed $24 \mathrm{~h}$ after completing the smoke exposure regimen to maximize the neutrophil population in the lung, and both the total number of BALF leukocytes and percentage of BALF neutrophils were measured (van der Vaart et al 2004). The protocol was approved by the institutional animal care committee.

\section{Treatment with LPS}

Prior to LPS instillation, animals were anesthetized by IP injection of pentobarbital $(90 \mathrm{mg} / \mathrm{kg})$. LPS was then delivered via the trachea with a 27 -gauge needle attached to a $1-\mathrm{mL}$ syringe. After instillation, the incision was closed with metal clips. The LPS used in these experiments was serotype O26:B6, obtained from American Type Culture Collection no. 12795; with short chain-length approximating that of mutant rough strain LPS.

\section{Exposure to cigarette smoke}

Animals were placed in a $70 \times 48 \times 38 \mathrm{~cm}$ chamber and passively exposed to cigarette smoke for $2 \mathrm{~h}$ per day for 3 consecutive days. The smoke was produced by a TE-10 smoking machine (Teague Enterprises, Davis, CA) that simultaneously burned 2 filtered research-grade cigarettes (type 2R4F, University of Kentucky). Smoke from both the filtered and burning ends of the cigarette were collected, then mixed together with air in a separate chamber, thus simulating second-hand smoke exposure. Each cigarette was puffed once per min for $2 \mathrm{sec}$ at a flow rate of $1.05 \mathrm{LPM}$, yielding $35 \mathrm{~mL}$ of smoke. This cycle was repeated 9 times before ejecting the cigarette and loading a new one. Proper flow rate was maintained by a vacuum pump that established negative pressure at the exhaust port. Total smoke particulates averaged $87 \mathrm{mg}$ per cubic meter.

\section{Bronchoalveolar lavage}

After euthanasia with i.p. pentobarbital, lungs were lavaged $3 \times$ with normal saline, using 1 aliquot of $3 \mathrm{~mL}$ followed by 2 aliquots of $2 \mathrm{~mL}$ each. The lavage fluid was centrifuged and the cells were resuspended in $1.0 \mathrm{~mL}$ PBS. Total leukocyte counts were determined with a hemocytometer. Differential counts were obtained on cytospin preparations treated with Wright's stain.

\section{Statistical analysis}

The Newman-Keuls multiple comparisons test was used to determine statistically significant differences $(p<0.05)$ among the treatment groups.

\section{Results LPS model}

Treatment with either ET-1 or Big ET-1 prior to LPS significantly increased the percentage of BALF neutrophils. As shown in Figure 1, animals receiving ET-1 or Big ET-1 had $89 \%$ and $80 \%$ neutrophils respectively, compared to $5 \%$ and $1 \%$ for controls treated only with intratracheal LPS or PBS, respectively. The remaining BALF cells in all treatment groups were predominantly macrophages.

Conversely, both ET-1 and Big ET-1 significantly lowered total BALF leukocytes in LPS-treated hamsters. Animals receiving ET-1 or Big ET-1 had $2.1 \times 10^{4}$ and $2.8 \times 10^{4}$ cells respectively, compared to $5.2 \times 10^{4}$ cells for those treated with intratracheal LPS alone (Figure 1). Controls given intratracheal PBS alone had $1.3 \times 10^{3}$ cells. 

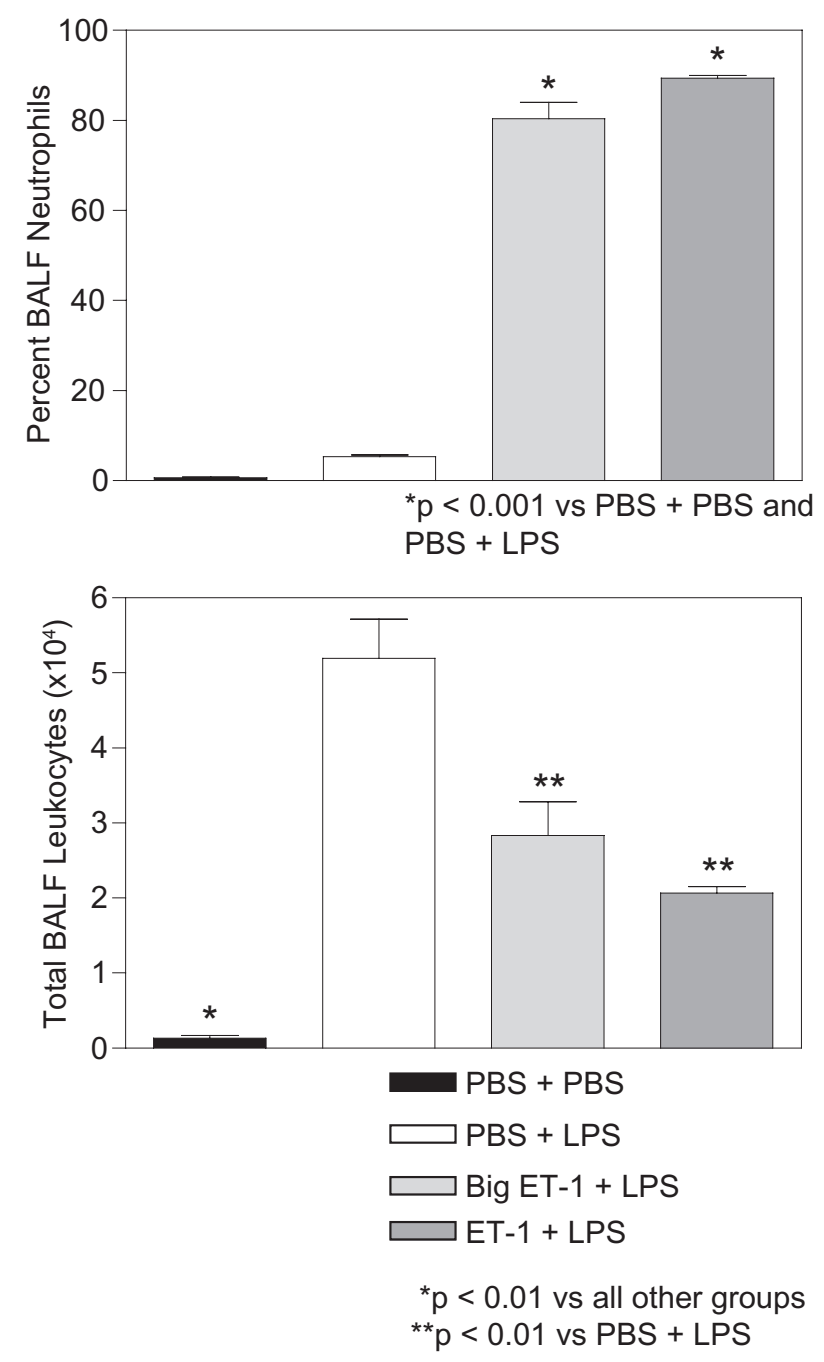

Figure I (Upper) Animals receiving ET-I or Big ET-I prior to LPS had a significantly higher percentage of BALF neutrophils than controls treated only with intratracheal LPS or PBS ( $\mathrm{N}=3$ for all groups). (Lower) Animals receiving ET-I or Big ET-I prior to LPS had significantly fewer total BALF leukocytes than controls treated with intratracheal LPS ( $\mathrm{N}=3$ for all groups). For both graphs, T-bars indicate standard error of the mean (SEM).

\section{Cigarette smoke model}

Treatment with ET-1 prior to exposure to cigarette smoke significantly increased the percentage of BALF neutrophils. As shown in Figure 2, hamsters receiving ET-1 had $46 \%$ neutrophils compared to $18 \%$ and $1 \%$ for controls treated only with cigarette smoke or ET-1 and room air, respectively. Furthermore, pretreatment with the ET-A receptor antagonist, HJP272, reversed this effect (Figure 2), lowering the percentage of BALF neutrophils to less than that of controls treated with cigarette smoke alone. The remaining BALF cells in all treatment groups were predominantly macrophages.

With regard to total BALF leukocytes, animals treated with either ET-1 or ET-1 plus HJP272 had fewer cells $\left(1.7 \times 10^{4}\right.$ and $1.3 \times 10^{4}$, respectively) than controls treated with cigarette smoke alone $\left(2.0 \times 10^{4}\right)$, but the differences among the groups were not statistically significant (Figure 2).

\section{Discussion}

The results of these experiments are consistent with selective recruitment of neutrophils by ET-1, and suggest that this mediator may play an important role in modulating the pulmonary inflammatory response. The level of ET-1 synthesis could determine whether the inflammatory cell population is predominantly composed of neutrophils or monocytes. Such variation in the nature of the inflammatory response could have important implications for the type and extent of lung damage resulting from exposure to different pulmonary toxins.

An example of the potential importance of modulating the pulmonary response to injury is seen in a previous study from this laboratory in which LPS and cigarette smoke were combined to produce a much greater inflammatory response than that seen with either agent alone (Kulkarni et al 2007). Whereas LPS and cigarette smoke separately produced a predominantly monocytic inflammatory cell influx into the lung, their combination resulted in a very large increase in recruitment of neutrophils to the lung, with concomitant enhancement of histopathological changes and markedly elevated alveolar septal cell apoptosis.

At the time this study was performed, the underlying mechanism for this transformation of the inflammatory response remained obscure. However, it is now possible to speculate that the dramatic rise in the neutrophil population could be related to enhanced synthesis of ET-1, whose effects might become much greater when a certain threshold level is achieved.

Of the two agents involved in the aforementioned study, LPS may be more effective in increasing ET-1 activity. Studies have shown that LPS stimulates the synthesis of ET-1 both in vivo and in vitro (Ohta et al 1990; Forni et al 2005; Zaedi et al 2006). Furthermore, the use of a nonselective endothelin receptor antagonist, tezosentan, has been shown to limit various hemodynamic abnormalities associated with endotoxemia, including pulmonary edema (Kuklin et al 2004; Rossi et al 2004).

The extent to which cigarette smoke enhances ET-1 synthesis may be directly related to LPS. In one study, cigarette smoke was found to contain 120 times the level of LPS associated with smoke-free indoor air (Larsson et al 2004). While this quantity of LPS may be small in comparison to that employed in the proposed experiments, it may nevertheless 

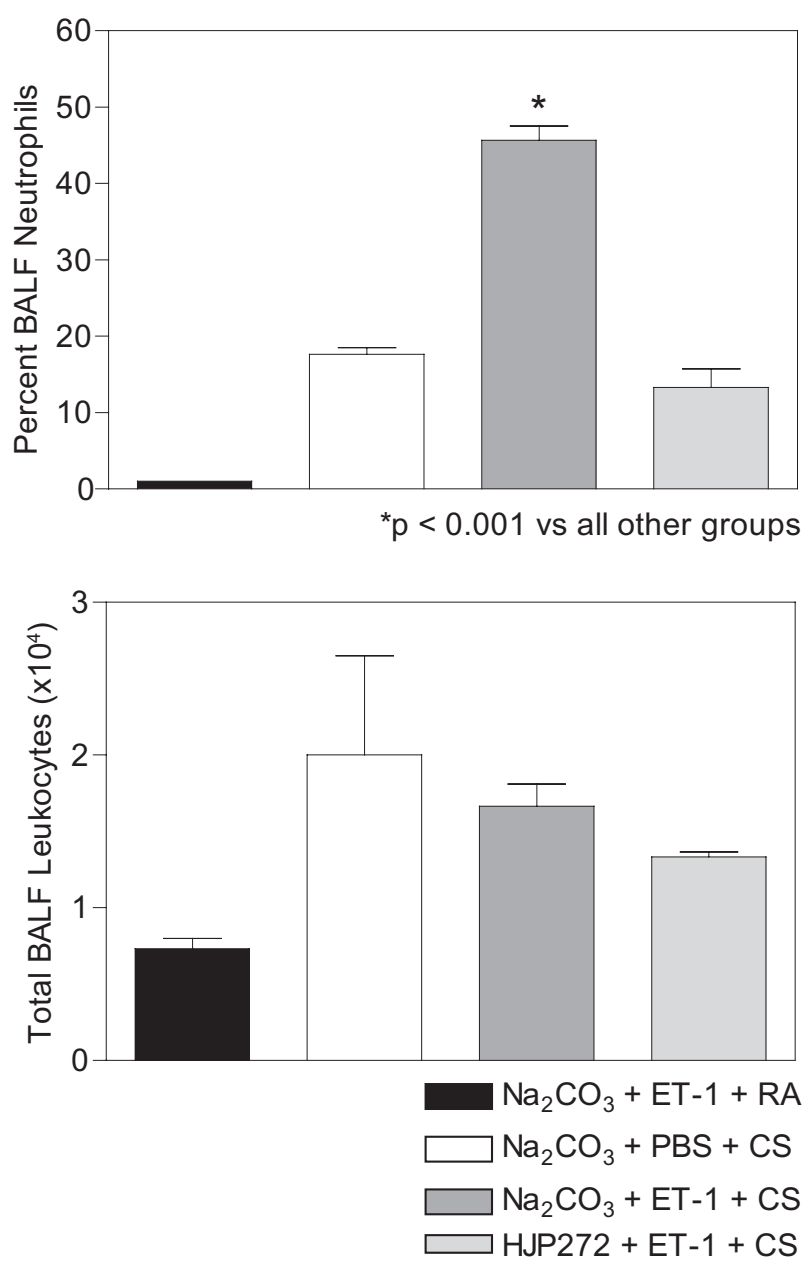

Figure 2 (Upper) Animals receiving ET-I prior to cigarette smoke (CS) exposure had a significantly higher percentage of BALF neutrophils than controls treated only with cigarette smoke or ET-I and room air (RA). The effect of ET-I was reversed by pretreatment with HJP272 ( $\mathrm{N}=3$ for all groups). (Lower) Animals receiving ET-I or ET-I plus HJP272 prior to CS had fewer total BALF leukocytes than controls treated with cigarette smoke alone, but the differences among these 3 treatment groups were not statistically significant ( $\mathrm{N}=3$ for all groups). For both graphs, T-bars indicate SEM.

have a cumulative effect, resulting in the development of lowgrade pulmonary inflammation that could cause significant lung damage over time.

While the mechanism responsible for the specific effect on neutrophils remains unclear, it may involve upregulation of ICAM-1, which facilitates their adhesion to vascular endothelium (Zhang et al 2006). Alternatively, ET-1 could increase neutrophil expression of CXCR2, a cell-surface receptor that binds interleukin-8, a potent activator of these cells (DiVietro et al 2001; Reutershan et al 2006). Either of these processes could be enhanced by the fact that ET-1 can change the F-actin content of neutrophils, thereby promoting their sequestration in pulmonary microvessels (Sato et al 2000).

In the current studies, treatment with an ET-A receptor antagonist reversed the effect of ET-1 on neutrophil recruitment to the lung. This finding may reflect an impaired ability of these cells to adhere to pulmonary vascular endothelium. Further support for this concept is found in studies of inflammatory bowel disease, where treatment with the nonselective endothelin receptor antagonist, bosentan, resulted in markedly reduced attachment of neutrophils to colonic submucosal venules (Anthoni et al 2006).

With regard to the cellular composition of inflammatory reactions, there is increasing evidence that ET-1 confers a selective advantage on neutrophils, allowing them to populate the lung at the expense of other inflammatory cells. Such a process might have important implications for the treatment of inflammatory lung disorders, including COPD. Reducing ET-1 activity with endothelin receptor antagonists and other agents could potentially decrease neutrophilderived enzymes and oxidants in the lung, thereby slowing the progression of lung injury.

\section{Acknowledgments}

This work was supported in part by grants from the Stony Wold - Herbert Fund and the Forest Research Institute.

\section{Disclosures}

The authors have no conflicts of interest to disclose.

\section{References}

Aguilar AM, Revert F, Moya A, et al. 2000. Intravenous BQ-123 and phosphoramidon reduce ventricular ectopic beats and myocardial infarct size in dogs submitted to coronary occlusion and reperfusion. Gen Pharmacol, 35:143-7.

Anthoni C, Mennigen RB, Rijcken EJ, et al. 2006. Bosentan, an endothelin receptor antagonist, reduces leucocyte adhesion and inflammation in a murine model of inflammatory bowel disease. Int J Colorectal Dis, 21:409-18.

Bhavsar T, Cerreta JM, Liu M, et al. 2008. Phosphoramidon, an endothelinconverting enzyme inhibitor, attenuates lipopolysaccharide-induced acute lung injury. Exp Lung Res, 34:1-14.

DiVietro JA, Smith MJ, Smith BRE, et al. 2001. Immobilized IL-8 triggers progressive activation of neutrophils rolling in vitro on p-selectin and intercellular adhesion molecule-1. J Immunol, 167:4017-25.

Fagan K, McMurtry I, Rodman D. 2001. Role of endothelin-1 in lung disease. Respir Res, 2:90-101.

Forni M, Mazzola S, Ribeiro LA, et al. 2005. Expression of endothelin-1 system in a pig model of endotoxic shock. Regul Pept, 131:89-96.

Hasday JD, Bascom R, Costa JJ, et al. 1999. Bacterial endotoxin is an active component of cigarette smoke. Chest, 115:829-35.

Hassan M, Kashimura H, Matsumaru K, et al. 1997. Phosphoramidon, an endothelin converting enzyme inhibitor attenuates local gastric ischemia-reperfusion injury in rats. Life Sci, 61:141-7.

Hisaki K, Matsumura Y, Maekawa H, et al. 1994. Conversion of Big ET-1 in the rat lung: role of phosphoramidon-sensitive endothelin-1-converting enzyme. Am J Physio, 266:H422-8.

Kuklin VN, Kirov MY, Evgenov OV, et al. 2004. Novel endothelin receptor antagonist attenuates endotoxin-induced lung injury in sheep. Crit Care Med, 32:766-73.

Kulkarni G, Nadkarni PP, Cerreta JM, et al. 2007. Short-term cigarette smoke exposure potentiates endotoxin-induced pulmonary inflammation. Exp Lung Res, 33:1-13. 
Laporte J, Sirois P. 1997. Phosphoramidon and thiorphan suppress the generation of endothelin (ET) from exogenous big-endothelin by guinea pig Clara cells. Regul Pept, 68:105-9.

Larsson 1, Szponar B, Pehrson C. 2004. Tobacco smoking increases dramatically air concentrations of endotoxin. Indoor Air, 14:421-4.

Nadkarni PP, Kulkarni GS, Cerreta JM, et al. 2005. Dichotomous effect of aerosolized hyaluronan in a hamster model of endotoxin-induced lung injury. Exp Lung Res, 31:807-18.

Ohta K, Hirata Y, Imai T, et al. 1990. Cytokine-induced release of endothelin-1 from porcine renal epithelial cell line. Biochem Biophys Res Commun, 169:578-84.

Park L, Thornhill J. 2001. Phosphoramidon-sensitive endothelin-converting enzymes modulate cerebral blood flow and neural damage of hypoxic rats. Neurosci Lett, 301:95-8.

Reutershan J, Morris MA, Burcin TL, et al. 2006. Critical role of endothelial CXCR2 in LPS-induced neutrophil migration into the lung. J Clin Invest, 116:695-702.
Rossi P, Wanecek M, Konrad D, et al. 2004. Tezosentan counteracts endotoxin-induced pulmonary edema and improves gas exchange. Shock, 21:543-8.

Sato Y, Hogg JC, English D, et al. 2000. Endothelin-1 changes polymorphonuclear leukocytes' deformability and CD11 b expression and promotes their retention in the lung. Am J Respir Cell Mol Biol, 23:404-10.

Teder P, Noble PW. 2000. A cytokine reborn? Endothelin-1 in pulmonary inflammation and fibrosis. Am J Respir Cell Mol Biol, 23:7-10.

Van der Vaart H, Postma DS, Timens W, et al. 2004. Acute effects of cigarette smoke on inflammation and oxidative stress: a review. Thorax, 59:713-21.

Zaedi S, Jesmin S, Maeda S, et al. 2006. Alterations in gene expressions encoding preproET-1 and NOS in pulmonary tissue in endotoxemic rats. Exp Biol Med, 231:992-6.

Zhang JS, Tan YR, Xiang Y, et al. 2006. Regulatory peptides modulate adhesion of polymorphonuclear leukocytes to bronchial epithelial cells through regulation of interleukins, ICAM-1 and NF-kappa B/lkappaB. Acta Biochim Biophys Sin, 38:119-28. 
